\title{
Relationship between the social support and self-efficacy for function ability in patients undergoing primary hip replacement
}

\author{
Kuan-Ting Wu ${ }^{1+}$, Pei-Shan Lee ${ }^{2 \dagger}$, Wen-Yi Chou ${ }^{1 *}$, Shu-Hua Chen ${ }^{2}$ and Yee-Tzu Huang ${ }^{3}$
}

\begin{abstract}
Background: The World Health Organization (WHO) reported that nearly 25\% of people will suffer from physical disability owing to the bone and joint problems until 2050. The condition of patients with this type of difficulty could be improved by increasing positive self-efficacy and instigating suitable medical treatment to implement self-efficacy for functional ability (SEFA) and physical functional ability self-care. In this study, we aim to evaluate the influence of social support on SEFA in patients after total hip arthroplasty.

Methods: This cross-sectional study used structural questionnaires, telephone appointments, and data collection to obtain patient characteristics, such as gender, age, educational level, and marital status. Questionnaires about social support and self-efficacy for functional ability (SEFA) were sent to 200 patients at 3 months following a primary total hip replacement from September 2011 to December 2014. Factor analysis was used to categorize the dimensions of social support; the $t$ test, analysis of variance (ANOVA), and correlation analysis were applied to screen factors influencing SEFA. Multiple regression analysis was employed to ascertain the relationships between patient characteristics, social support, and SEFA.

Results: In total, 134 patients responded to the questionnaires. Lower SEFA scores were observed in patients of an older age, unmarried patients, and those with a low level of education. Correlation analysis showed that emotional information and appraisal support, instrumental support, and SEFA were positively correlated. Multiple regression analysis was applied to ascertain the relationships between patient characteristics, social support, and SEFA. We identified significant coefficient values of -0.187 for age, 5.344 for emotional information and appraisal support, and 1.653 for instrumental support.

Conclusion: The results of this study demonstrated that in patients undergoing primary hip replacement, positive impacts on SEFA were observed in relation to emotional information, appraisal support and instrumental support. The results indicated that enhancing emotional information and appraisal support could improve a patient's selfefficacy for functional ability.
\end{abstract}

Keywords: Self-efficacy for functional ability, Social support, Primary hip replacement, Arthroplasty

\footnotetext{
*Correspondence: murraychou@yahoo.com.tw

${ }^{+}$Kuan-Ting Wu and Pei-Shan Lee contributed equally to this work.

'Department of Orthopaedic Surgery, Kaohsiung Chang Gung Memorial

Hospital, No.123, Dapi Rd., Niaosong Dist., Kaohsiung city 833, Taiwan,

Republic of China

Full list of author information is available at the end of the article
}

(c) The Author(s). 2018 Open Access This article is distributed under the terms of the Creative Commons Attribution 4.0 International License (http://creativecommons.org/licenses/by/4.0/), which permits unrestricted use, distribution, and reproduction in any medium, provided you give appropriate credit to the original author(s) and the source, provide a link to the Creative Commons license, and indicate if changes were made. The Creative Commons Public Domain Dedication waiver (http://creativecommons.org/publicdomain/zero/1.0/) applies to the data made available in this article, unless otherwise stated. 


\section{Background}

Degenerative hip disease is the most common form of hip discomfort. According to the World Health Organization (WHO), bone and joint diseases may result in physical disability in nearly 25\% of the population until 2050. Degenerative osteoarthritis can be diagnosed via physical examination in almost $80 \%$ of patients older than 55-65 years, and 20\% of these patients already have a disability [1]. Based on research conducted by the Centers for Disease Control and Prevention (CDC) in the USA, the number of patients with osteoarthritis will double in the population over 65 years of age by 2030 [2].

Degenerative osteoarthritis often results in pain and limitation of daily activity, which are the leading causes requiring medical assistance [3]. Initially, these discomforts can be alleviated by appropriate medical management and self-care modification. In addition, caregivers play a key role in psychological support by providing patients with company [4]. In patients who are refractory to conservative treatment, a total hip replacement (THR) is usually indicated. According to the literature, THR yields excellent pain relief, with a survival rate from 90 to $99.8 \%$ during a 10-15-year follow-up period [5-7]. However, functional outcome varies according to preoperative characteristics, such as age, comorbidities, and activity level [8]. In addition to surgical technique, postoperative rehabilitation, activity adjustment, and posture limitation are also important for better functional recovery. Psychosocial factors are also associated with recovery after THR [9, 10].

Pain from degenerative osteoarthritis has a negative effect on patients in terms of psychosocial issues in the long term, such as effects on mood and sleep, which interact with activity level [11]. Langford and Bowsher [12] pointed out that adequate social support has positive effects on health status and behavior. In patients with a hip fracture, social support is also correlated with the function of the lower limbs [13]. Self-efficacy represents a patient's personal confidence in completing specific tasks and also brings about a sense of accomplishment. After lower limb arthroplasty, Moon and Backer identified significant correlations between self-efficacy, walking distance, and lower limb activity frequency [14]. In the elderly population, health status and physical function are key factors affecting the execution of regular exercise; therefore, the condition of patients with this type of difficulty may be improved by increasing positive self-efficacy and social support following THR [15].

In this study, we aimed to examine the relationships between social support, self-efficacy level, and functional ability in patients undergoing primary THR after discharge from the hospital.

\section{Methods}

\section{Study design and sample}

This cross-sectional study used structural questionnaires, telephone appointments, and data collection to obtain patient characteristics, such as gender, age, educational level, and marital status. The Institutional Review Board at our hospital approved this analysis. Patients who had undergone THR due to osteoarthritis of the hip were enrolled from September 2011 to December 2014. Patients with the following conditions were excluded: dementia, psychological disorders, or postoperative complications such as cardiovascular accident, fat embolism, vascular or nerve injury, intraoperative acetabulum, or femur fracture. We used modified versions of the self-efficacy for functional ability (SEFA) scale and the social support scale for patient evaluation.

\section{Questionnaires}

We collected patient characteristics such as gender, age, educational level, and marital status. Self-efficacy for functional ability (SEFA) and social support questionnaires were completed by the patients 3 months following primary THR. As the average age of the patients was greater than 65 years, we used modified versions of the questionnaires for simplification.

\section{Social support scale}

The modified social support scale [16] was revised from the Inventory of Socially Supportive Behaviors (ISSB) developed by Barrera et al. [17] for the measurement of assistance provided in the areas of emotional information, appraisal support, and instrumental support by a spouse, family, friends, and relatives. The patients were asked to respond to 15 items using a Likert scale from 0 to 3 points, representing no assistance, little assistance, much assistance, and very much assistance, respectively. Hence, the global score ranged from 0 to 90, with higher scores indicating better social support. In addition, we categorized social support into two dimensions. Dimension 1 represented emotional information and appraisal support, with a Cronbach's alpha of 0.973, while dimension 2 represented instrumental support, with a Cronbach's alpha of 0.767 .

\section{Self-efficacy for functional ability scale}

The SEFA scale is usually employed to assess patient confidence in performing daily activities. In this study, we used a modified SEFA proposed by Resnick [18]. Nine items were included in the modified SEFA questionnaire, which assessed functional ability in terms of dressing, getting out of and into bed, using the toilet, bathing, walking, and climbing stairs. Items were scored from 1 to 4 , representing patient levels of confidence of less than $20 \%, 20-50 \%, 50-80 \%$, and greater than $80 \%$, 
respectively, in performing the activities by themselves. The total score ranged from 9 to 36, with higher scores representing greater confidence in performing daily activities. The Cronbach's alpha for this scale was 0.969 .

\section{Statistical analysis}

Factor analysis was used to categorize dimensions of social support. The Kaiser-Meyer-Olkin value was 0.898, and the Barlett test showed a $p$ value $<0.001$. Furthermore, the $t$ test, analysis of variance (ANOVA), and correlation analysis were applied to screen the factors influencing SEFA. Multiple regression analysis was employed to ascertain the relationships between patient characteristics, social support, and SEFA. The reliability of the questionnaires was assessed using Cronbach's alpha; the higher the Cronbach's alpha value, the greater the internal consistency.

\section{Results}

From September 2011 to December 2014, 200 patients underwent THR for advanced osteoarthritis. Following the exclusion of 66 patients who met the exclusion criteria, 134 patients were enrolled in this study, $51.5 \%$ of whom were male; $21.6 \%$ of the patients were under 55 years of age, $23.1 \%$ were aged between 51 and 64 , $35.1 \%$ between 65 and 75 , and $20.1 \%$ were over 75 years of age. $38.1 \%$ of the patients had an educational level ranging from elementary school to junior high school, and $26.9 \%$ were uneducated. $86.6 \%$ of the patients were married (Table 1).

In terms of social support, the average score for the dimension of emotional information and appraisal support was $1.81 \pm 0.7$, while that for the dimension of

Table 1 Characteristics of primary THR patients $(n=134)$

\begin{tabular}{lll}
\hline & Number & Percentage (\%) \\
\hline Sex & 69 & 51.5 \\
Male & 65 & 48.5 \\
$\quad$ Female & & \\
Age (years) & 29 & 21.6 \\
$<51$ & 31 & 23.1 \\
$51-64$ & 47 & 35.1 \\
$65-75$ & 27 & 20.1 \\
$>75$ & & \\
Educational level & 36 & 26.9 \\
Uneducated & 51 & 38.1 \\
Primary/secondary school & 47 & 35.1 \\
$>$ Senior high school & & \\
Marital status & 18 & 86.6 \\
Single & 116 & \\
Married & &
\end{tabular}

instrumental support was $1.56 \pm 0.77$. The highest score $(2.08 \pm 0.65)$ of the items in the dimension of emotional information and appraisal support was obtained for "expressed interest and concern in your well-being," followed by "reminded you of follow-up hospital appointments" (2.02 \pm 0.77$)$. Furthermore, the lowest score was obtained for the item "assisted you in appropriate exercise" $(1.37 \pm 0.85)$. In the dimension of instrumental support, the lowest score $(1.50 \pm 0.75)$ was obtained for the item "listened to you talk about your private feelings" (Table 2).

The average SEFA score of the patients after THR was $3.33 \pm 0.84$. The lowest-scored items in terms of self-efficacy were "wash your lower body $(3.24 \pm 0.95)$," "dress your lower body (3.19 \pm 1.00$)$," and "climb up and down four stairs $(2.78 \pm 1.21)$." The highest score (3.53 \pm 0.28 ) was obtained for "get in and out of bed and chairs" (Table 3). Male patients scored $19.97 \pm 8.21$ and female patients $21.95 \pm 6.84$ on average, with a $p$ value $<0.01$.

A positive correlation between emotional information and appraisal support with instrumental support was observed, with a Pearson correlation coefficient of 0.6. In addition, a positive relationship between emotional information and appraisal support with SEFA was seen, with a Pearson correlation coefficient of 0.635 (Table 4).

Scores on the scales of self-efficacy $(17.58 \pm 8.98)$ and emotional information and appraisal support $(1.46 \pm 0.80)$ were lower in the uneducated patients $(p<0.01)$, as was the instrumental support score $(p<0.05)$. In addition, the elderly patients had lower SEFA scores $(p<0.01)$ (Table 5).

We included predictors selected from $t$ tests and ANOVA, such as sex, age, educational level, marital status, emotional information, appraisal support, and instrumental support in stepwise multivariable regression analysis. After the elimination procedure, age, educational level, emotional information and appraisal support, and instrumental support were selected as significant predictors. These significant predictors were analyzed using an all-possible regression procedure. It was found that an older age was related to a lower self-efficacy $(b=-0.187, p<0.001)$. Emotional information and appraisal support had a positive influence on self-efficacy $(b=5.344, p<0.001)$, as did instrumental support $(b=1.653, p<0.05)$. The model explained approximately $52.6 \%$ of the variance in SEFA 3 months after THR, and the final regression model explained $50.7 \%$ of the variance (Table 6).

\section{Discussion}

Social support is a key factor related to the daily activity of geriatric patients. Friedland and McColl [19] pointed out that interventions to increase social support in critical patients can improve chronic stress and physical 
Table 2 Descriptive analysis of social support after THR

\begin{tabular}{|c|c|c|c|c|c|}
\hline & No assistance (0) & Little & Much & Very much & Mean \pm SD \\
\hline & Number (\%) & & & & \\
\hline Emotional information and appraisal support & & & & & $1.81 \pm 0.70$ \\
\hline Expressed interest and concern in your well-being & $0(0 \%)$ & $23(17 \%)$ & $78(57.8 \%)$ & $34(25.2 \%)$ & $2.08 \pm 0.65$ \\
\hline Was right there with you in a stressful situation & $5(3.7 \%)$ & $32(23.7 \%)$ & $76(56.3 \%)$ & $22(16.3 \%)$ & $1.85 \pm 0.73$ \\
\hline Joked and kidded to try to cheer you up & $4(3 \%)$ & $23(17 \%)$ & $82(60.7 \%)$ & $26(19.3 \%)$ & $1.96 \pm 0.69$ \\
\hline Agreed that what you want to do was right & $8(6 \%)$ & $35(13.4 \%)$ & $73(26.1 \%)$ & $18(54.5 \%)$ & $1.75 \pm 0.76$ \\
\hline Let you know you did something well & $8(6 \%)$ & $25(18.7 \%)$ & $34(25.4 \%)$ & $67(50 \%)$ & $1.81 \pm 0.80$ \\
\hline $\begin{array}{l}\text { Expressed esteem or respect for a competency } \\
\text { or personal quality of yours }\end{array}$ & $6(4.5 \%)$ & $29(21.6 \%)$ & $32(23.9 \%)$ & $67(50 \%)$ & $1.93 \pm 0.79$ \\
\hline Provided some advice on medical treatment & $8(6 \%)$ & $30(22.4 \%)$ & $31(23.1 \%)$ & $65(48.5 \%)$ & $1.87 \pm 0.82$ \\
\hline Provided some advice on daily living & $6(4.5 \%)$ & $25(18.7 \%)$ & $36(26.9 \%)$ & $67(50 \%)$ & $1.83 \pm 0.78$ \\
\hline Reminded you of follow-up hospital appointments & $6(4.5 \%)$ & $20(14.9 \%)$ & $35(26.1 \%)$ & $73(54.4 \%)$ & $2.02 \pm 0.77$ \\
\hline Assisted you in setting a goal for yourself & $13(9.7 \%)$ & $26(19.4 \%)$ & $47(35.1 \%)$ & $48(35.8 \%)$ & $1.65 \pm 0.90$ \\
\hline $\begin{array}{l}\text { Did some activity together to help you get your minds } \\
\text { off things }\end{array}$ & $24(17.9 \%)$ & $26(19.4 \%)$ & $27(20.1 \%)$ & $57(42.5 \%)$ & $1.59 \pm 0.99$ \\
\hline Helped you in adjustment of daily activities & $8(6 \%)$ & $32(23.9 \%)$ & $42(31.3 \%)$ & $52(38.8 \%)$ & $1.81 \pm 0.87$ \\
\hline Assisted you in appropriate exercise & $12(9 \%)$ & $21(15.7 \%)$ & $46(34.3 \%)$ & $55(41 \%)$ & $1.37 \pm 0.58$ \\
\hline Instrumental support & & & & & $1.56 \pm 0.77$ \\
\hline Listened to you talk about your private feelings & $9(6.7 \%)$ & $62(45.9 \%)$ & $52(38.5 \%)$ & $12(8.9 \%)$ & $1.50 \pm 0.75$ \\
\hline Loaned or gave you something that you needed & $14(10.4 \%)$ & $32(23.9 \%)$ & $34(25.4 \%)$ & $54(40.3 \%)$ & $1.63 \pm 0.96$ \\
\hline
\end{tabular}

disability. Social support also has positive effects on health status and behavior [12] and can enhance execution of healthy behaviors, such as taking medicines on time, engaging in regular exercise, and diet control [20]. In studies of patients with hip fractures, a significant relationship was also identified between social support and lower limb functional activity [13], especially when supported by their spouse and family [21]. Social support is usually provided by the patient's family members who live with them. Kiefer identified a higher level of social support in patients living with a spouse, adult child, or friends while the singles had a lower level of social support [22]. In this study, we found that the item with the highest score $(2.08 \pm 0.65)$ was "expressed interest and concern in your well-being." Because it is easier for a caregiver or family to focus on the patient's disease-related health status, as they can directly observe their gait, muscle power, and agility following THR. Sveikata et al. [23] demonstrated better postoperative functional results 12 months after total knee arthroplasty in patients who had better social support. McHugh et al. [24] also identified key psychosocial factors and

Table 3 Descriptive analysis of SEFA after THR

\begin{tabular}{|c|c|c|c|c|c|}
\hline \multirow{2}{*}{$\begin{array}{l}\text { Confidence (score) } \\
\text { Item }\end{array}$} & $<20 \%(1)$ & \multirow{2}{*}{$\begin{array}{l}20-50 \% \\
(2)\end{array}$} & \multirow{2}{*}{$\begin{array}{l}50-80 \% \\
\text { (3) }\end{array}$} & \multirow[t]{2}{*}{$>80 \%(4)$} & \multirow[t]{2}{*}{ Mean \pm SD } \\
\hline & Number (\%) & & & & \\
\hline Wash your upper body & $8(6 \%)$ & $10(7.5 \%)$ & $28(20.9 \%)$ & $88(65.7 \%)$ & $3.46 \pm 0.87$ \\
\hline Wash your lower body & $9(6.7 \%)$ & $21(15.7 \%)$ & $33(24.6 \%)$ & $71(53 \%)$ & $3.24 \pm 0.95$ \\
\hline Dress your upper body & $4(3 \%)$ & $12(9 \%)$ & $26(19.4 \%)$ & $92(68.7 \%)$ & $3.48 \pm 0.92$ \\
\hline Dress your lower body & $9(6.7 \%)$ & $24(17.9 \%)$ & $29(21.6 \%)$ & $72(53.7 \%)$ & $3.19 \pm 1.00$ \\
\hline Get on and off the toilet and manage your clothes & $8(6 \%)$ & $13(9.7 \%)$ & $20(14.9 \%)$ & $93(69.4 \%)$ & $3.48 \pm 0.90$ \\
\hline Get in and out of bed and a chair & $4(3 \%)$ & $8(6 \%)$ & $31(23.1 \%)$ & $91(67.9 \%)$ & $3.53 \pm 0.82$ \\
\hline Walk $50 \mathrm{ft}$ & $4(3 \%)$ & $12(9 \%)$ & $32(23.9 \%)$ & $86(64.2 \%)$ & $3.43 \pm 0.92$ \\
\hline Walk $120 \mathrm{ft}$ & $6(4.5 \%)$ & $12(9 \%)$ & $37(27.6 \%)$ & $79(59 \%)$ & $3.37 \pm 0.93$ \\
\hline Climb up and down four stairs & $22(16.4 \%)$ & $26(19.4 \%)$ & $31(23.1 \%)$ & $55(41 \%)$ & $2.78 \pm 1.21$ \\
\hline Total score & & & & & $29.96 \pm 7.6$ \\
\hline Total average score & & & & & $3.33 \pm 0.84$ \\
\hline
\end{tabular}


Table 4 Pearson's correlations with SEFA scale

\begin{tabular}{lll}
\hline Variable & $\begin{array}{l}\text { Correlation } \\
\text { coefficient, } r\end{array}$ & $p$ value \\
\hline $\begin{array}{l}\text { Emotional information } \\
\text { and appraisal support }\end{array}$ & 0.635 & $<0.001$ \\
Instrumental support & 0.489 & $<0.001$ \\
\hline
\end{tabular}

biomedical predictors of pain, anxiety, and depression related to recovery following THR in 206 patients. Social support can affect self-efficacy in terms of verbal encouragement. In a randomized control study, improved general self-efficacy and physical function were observed in the group who received telephone follow-up appointments conducted by a nurse, which was structured along the lines of the VIPS model (the Swedish acronym for the concepts of Well-being, Integrity, Prevention and Safety) [25]. We also identified a significant positive correlation between social support and self-efficacy in our study, with a coefficient of 5.344 for emotional information and appraisal support and 1.653 for instrumental support.

Physical activity is crucial in the elderly and can prevent chronic disease, promote physical health, and help to maintain the quality of life [26]. Elderly persons who engage in regular exercise will perform better in functions of daily activity and will have an improved health status and reduce few chronic diseases such as stroke, cardiovascular disease, osteoporosis, hypertension, and diabetes [27, 28]. If patients are restricted in terms of the execution of physical activity, they will be unwilling to perform daily activities [29]. Similar with social support, increase self-efficacy is associated with higher physical activity levels [30, 31]. Poor self-efficacy usually leads to greater distress in patients during their recovery; they often worry about their condition getting worse after executing a specific task because they are not confident in their course of rehabilitation. In research into self-efficacy, self-efficacy was taken as an evaluation of capability in terms of achievement in specific tasks, a significant correlation between self-efficacy and personal success was identified [32, 33]. Dominick et al. demonstrated a significant correlation between low self-efficacy for exercise and less improvement in functional recovery over time after total knee arthroplasty [34]. Waldrop et al. [35] also addressed self-efficacy predicted significant variance in rehabilitation outcomes in orthopedic surgery. They concluded that augmenting self-efficacy beliefs by psychologists could improve functional recovery. The higher the level of induced self-efficacy, the greater the performance accomplishments.

Patients who undergo THR are usually discharged home directly. Therefore, it is easy to assess the correlation of functional outcome with the quality of home care. Self-efficacy was identified as a significant predictor of recovery after joint arthroplasty by Moon and Backer et al. [14]. They also found that walking distance and the

Table 5 Group comparison of emotional information and appraisal support, instrumental support and SEFA

\begin{tabular}{|c|c|c|c|c|c|c|}
\hline \multirow[t]{2}{*}{ Variable } & \multicolumn{2}{|c|}{ Emotional information and appraisal support } & \multicolumn{2}{|c|}{ Instrumental support } & \multicolumn{2}{|c|}{ Self-efficacy of functional ability } \\
\hline & $\mathrm{M} \pm \mathrm{SD}$ & $t, F$ value & $\mathrm{M} \pm \mathrm{SD}$ & $t, F$ value & $\mathrm{M} \pm \mathrm{SD}$ & $t, F$ value \\
\hline $\operatorname{Sex}(T)$ & & 4.454 & & $0.563^{* *}$ & & $3.487^{* *}$ \\
\hline Male & $1.66 \pm 0.75$ & & $1.35 \pm 0.73$ & & $19.91 \pm 8.21$ & \\
\hline Female & $1.97 \pm 0.61$ & & $1.80 \pm 0.75$ & & $21.95 \pm 6.84$ & \\
\hline Age (years)(A) & & 0.954 & & 0.197 & & $8.123^{* *}$ \\
\hline$(1)<50$ & $1.88 \pm 0.74$ & & $1.64 \pm 0.91$ & & $23.83 \pm 4.65$ & \\
\hline (2) $51-64$ & $1.74 \pm 0.56$ & & $1.48 \pm 0.46$ & & $21.27 \pm 9.18$ & \\
\hline (3) $65-75$ & $1.90 \pm 0.68$ & & $1.55 \pm 0.79$ & & $22.10 \pm 4.63$ & \\
\hline (4) $>75$ & $1.64 \pm 0.83$ & & $1.58 \pm 0.91$ & & $15.04 \pm 9.74$ & \\
\hline Post hoc Scheff & & & & & $(4)<(1),(2),(3)$ & \\
\hline Educational level (A) & & $8.978^{* *}$ & & $4.430^{*}$ & & $7.476^{*}$ \\
\hline (1) Uneducated & $1.46 \pm 0.80$ & & $1.39 \pm 0.73$ & & $17.58 \pm 9.00$ & \\
\hline (2) Primary to secondary school & $2.07 \pm 0.50$ & & $1.81 \pm 0.73$ & & $23.65 \pm 4.97$ & \\
\hline (3) $>$ Senior high school & $1.79 \pm 0.72$ & & $1.43 \pm 0.80$ & & $20.12 \pm 8.16$ & \\
\hline Post hoc Scheff & $(1)<(2)$ & & $(1)<(2)$ & & $(1)<(2)$ & \\
\hline Marital status (T) & & 3.502 & & 0.284 & & $8.182^{*}$ \\
\hline Single & $1.88 \pm 0.46$ & & $1.64 \pm 0.68$ & & $20.51 \pm 7.90$ & \\
\hline Married & $1.80 \pm 0.73$ & & $1.55 \pm 0.79$ & & $23.83 \pm 4.40$ & \\
\hline
\end{tabular}

A represents ANOVA and T represents independent $t$ test ${ }^{*} p<0.05^{*},{ }^{* *} p<0.01$ 
Table 6 Multivariable regression analysis of SEFA

\begin{tabular}{|c|c|c|c|}
\hline SEFA & & & \\
\hline Variable & B & $S(B)$ & VIF \\
\hline \multicolumn{4}{|l|}{ Patient characteristics } \\
\hline Age & $-\overline{0.187^{* * *}}$ & $-\overline{0} \cdot 333$ & 1.355 \\
\hline \multicolumn{4}{|l|}{ Educational level } \\
\hline \multicolumn{4}{|l|}{ Uneducated (reference) } \\
\hline Primary/secondary school & -0.039 & $-\overline{0}-003$ & 2.038 \\
\hline$>$ Senior high school & -2.644 & $-\overline{0} 161$ & 1.875 \\
\hline \multicolumn{4}{|l|}{ Social support } \\
\hline $\begin{array}{l}\text { Emotional information and appraisal } \\
\text { support }\end{array}$ & $5.344^{* * *}$ & 0.491 & 1.714 \\
\hline Instrumental support & $1.653^{*}$ & 0.166 & 1.609 \\
\hline
\end{tabular}

frequency of lower limb activity increased as self-efficacy improved. In our study, the SEFA item with the highest score was "get in and out of bed and chairs," while the lowest-scored item was "climb up and down four stairs". As climbing stairs requires high levels of coordination and strength, it is difficult for patients to complete this task with ease. Self-efficacy might be affected in several ways. According to the literature, there exist two factors that influence the daily activity and self-efficacy of patients who have undergone THR: patient characteristics and disease characteristics. In terms of patient characteristics, confidence in self-efficacy decreases with aging [36, 37]. Janiszewska et al. demonstrated that older age deteriorated general health and decreased general self-efficacy level in women who undergone osteoporosis treatment [38]. With regard to the level of education, Lien and Wei studied 350 patients separated into diabetic and non-diabetic groups, and a better self-efficacy was associated with a higher educational level [39]. Marital status also affects self-efficacy in correlation with social support. Fitzgerald et al. [40] evaluated 222 patients 12 months after joint replacement surgery, and better social support and self-efficacy were noted in patients who were married or living with someone. Regarding disease characteristics, patients have difficulties in performing daily activities owing to specific posture limitations during the first 6 to 12 weeks following THR [41, 42]. In our study, we evaluated patients 3 months following THR in order to minimize bias and observed better SEFA in married patients and those with a higher level of education.

Some studies have identified different contributions of preoperative and postoperative self-efficacy to patient outcome following THR [43-45]. A higher preoperative self-efficacy means that the patient has more confidence in their recovery and is willing to undergo THR; furthermore, preoperative self-efficacy has also been identified as a predictor of better recovery. However, in a systemic review regarding the influence of self-efficacy on functional recovery, the effect of preoperative self-efficacy was inconclusive, while in contrast, the effect of postoperative self-efficacy was consistent and was found to be associated with functional recovery in terms of distance ambulation, exercise repetition, and frequency [45, 46]. Some studies have indicated that self-efficacy is associated with emotional outcomes [47]. In our study, we attempted to identify factors correlated with postoperative SEFA; therefore, we evaluated the relationships of emotional information and instrumental support with postoperative SEFA and identified significant correlations, with Pearson coefficients of 0.635 and 0.483 , respectively.

\section{Limitations}

First, we used modified questionnaires in order to make it easier for the elderly participants to respond to items. The validity of the SEFA and social support questionnaires was examined, and both had a high internal consistency, with Cronbach's alpha values greater than 0.7 ; however, these values were still lower than those of the primary versions of the questionnaires. Second, all patients were enrolled from a single hospital, and differences in culture in other cities might result in differences in social support and self-efficacy.

\section{Conclusion}

This study concluded that in patients undergoing primary hip replacement, emotional information, appraisal support, and instrumental support had positive impacts on self-efficacy for functional ability. The results indicated that enhancing emotional information and appraisal support could improve self-efficacy for functional ability and lead to a better recovery following THR.

\section{Abbreviations}

CDC: Centers for Disease Control and Prevention; SEFA: Self-efficacy for functional ability; THR: Total hip replacement; WHO: World Health Organization

\section{Acknowledgements}

We would like to acknowledge Y. H Chuang for formatting the draft and Chang Gung Statistical Analysis Center for the counsel.

\section{Availability of data and materials}

The data supporting the conclusion of this study is included in the manuscript and Table 6. All data generated or analyzed during this study are included in this published article.

\section{Authors' contributions}

PSL contributed to the planning of the study, recruitment of the patients, data interpretation, writing of the draft, and literature review and revised and analyzed the data and statistics. KTW contributed to the data interpretation, writing of the draft, literature review, and critical revision of the paper and revised and analyzed the data and statistics. YTH contributed to the data 
interpretation and writing of the draft and revised and analyzed the data and statistics. SHC organized the study and contributed to the clinical analysis of the data and critical commenting and improvement of the manuscript. WYC contributed to the critical revision of the paper and submitted revisions. All authors read and approved the final manuscript. PSL and KTW contributed equally to the article and listed as the co-first author.

\section{Ethics approval and consent to participate}

This retrospective study was approved by the Institutional Review Board (IRB) of Chang Gung Medical Foundation (IRB Study No. 100-1344C). Written informed consent to participate in this study was obtained from the participants.

\section{Competing interests}

The authors declared that they have no competing interests.

\section{Publisher's Note}

Springer Nature remains neutral with regard to jurisdictional claims in published maps and institutional affiliations.

\section{Author details}

'Department of Orthopaedic Surgery, Kaohsiung Chang Gung Memorial Hospital, No.123, Dapi Rd., Niaosong Dist., Kaohsiung city 833, Taiwan, Republic of China. ${ }^{2}$ Department of Orthopedics Operation Room, Kaohsiung Chang Gung Memorial Hospital, No.123, Dapi Rd., Niaosong Dist., Kaohsiung city 833, Taiwan, Republic of China. ${ }^{3}$ Department of Hospital and Health Care Administration, Chia Nan University of Pharmacy and Science, No.60, Sec. 1, Erren Rd., Rende Dist, Tainan city 717, Taiwan, Republic of China.

\section{Received: 20 April 2018 Accepted: 5 June 2018}

\section{Published online: 18 June 2018}

\section{References}

1. Baird CL, Schmeiser D, Yehle KT. Self-caring of women with osteoarthritis living at different levels of independence. Health Care Women Int. 2003;24: 617-34

2. Public health and aging. projected prevalence of self-reported arthritis or chronic joint symptoms among persons aged $>65$ years-United States, 2005-2030. MMWR Morb Mortal Wkly Rep. 2003;52:489-91.

3. Murphy L, Helmick CG. The impact of osteoarthritis in the United States: a population-health perspective: a population-based review of the fourth most common cause of hospitalization in US adults. Orthop Nurs. 2012;31: $85-91$.

4. Chen $\mathrm{Y}-\mathrm{C}$, Yuan S-C. The effectiveness of an intervention program among total hip replacement patients. Chung Shan Medical Journal. 2003:14:109-18.

5. Williams HD, Browne G, Gie GA, Ling RS, Timperley AJ, Wendover NA. The Exeter universal cemented femoral component at 8 to 12 years. A study of the first 325 hips. J Bone Joint Surg Br. 2002;84:324-34.

6. Clohisy JC, Harris WH. The Harris-Galante porous-coated acetabular component with screw fixation. An average ten-year follow-up study. J Bone Joint Surg Am. 1999;81:66-73.

7. Lee YK, Ha YC, Yoo JJ, Koo KH, Yoon KS, Kim HJ. Alumina-on-alumina total hip arthroplasty: a concise follow-up, at a minimum of ten years, of a previous report. J Bone Joint Surg Am. 2010;92:1715-9.

8. Fortin PR, Penrod JR, Clarke AE, St-Pierre Y, Joseph L, Belisle P, et al. Timing of total joint replacement affects clinical outcomes among patients with osteoarthritis of the hip or knee. Arthritis Rheum. 2002;46:3327-30.

9. Keefe FJ, Smith SJ, Buffington AL, Gibson J, Studts JL, Caldwell DS. Recent advances and future directions in the biopsychosocial assessment and treatment of arthritis. J Consult Clin Psychol. 2002;70:640-55.

10. Brembo EA, Kapstad H, Van Dulmen S, Eide H. Role of self-efficacy and social support in short-term recovery after total hip replacement: a prospective cohort study. Health Qual Life Outcomes. 2017;15:68.

11. Neogi T. The epidemiology and impact of pain in osteoarthritis. Osteoarthr Cartil. 2013;21:1145-53.

12. Langford CP, Bowsher J, Maloney JP, Lillis PP. Social support: a conceptual analysis. J Adv Nurs. 1997;25:95-100.

13. Mock C, MacKenzie E, Jurkovich G, Burgess A, Cushing B, deLateur B, et al. Determinants of disability after lower extremity fracture. J Trauma. 2000;49: 1002-11.
14. Moon $L B$, Backer J. Relationships among self-efficacy, outcome expectancy, and postoperative behaviors in total joint replacement patients. Orthop Nurs. 2000;19:77-85.

15. Struck BD, Ross KM. Health promotion in older adults. Prescribing exercise for the frail and home bound. Geriatrics. 2006;61:22-7.

16. Wang YH. Study on life quality and its associated factors of rheumatoid arthritis patients. Journal of Chang Gung Institute of Nursing. 2000;2:71-95.

17. Barrera M, Sandler IN, Ramsay TB. Preliminary development of a scale of social support: studies on college students. Am J Community Psychol. 1981;9:435-47.

18. Resnick B. Efficacy beliefs in geriatric rehabilitation. J Gerontol Nurs. 1998;24: 34-44.

19. Friedland JF, McColl M. Social support intervention after stroke: results of a randomized trial. Arch Phys Med Rehabil. 1992;73:573-81.

20. Chiang W-Y, Chung H-H. Hemodialysis patients' fatigue relating to depression, social support and blood biochemical data. The Journal of Nursing Research. 1997;5:115-26.

21. Saltz CC, Zimmerman S, Tompkins C, Harrington D, Magaziner J. Stress among caregivers of hip fracture patients. J Gerontol Soc Work. 1999;30:167-81.

22. Kiefer RA. The effect of social support on functional recovery and wellbeing in older adults following joint arthroplasty. Rehabil Nurs. 2011;36:120-6.

23. Sveikata T, Porvaneckas N, Kanopa P, Molyte A, Klimas D, Uvarovas V, et al. Age, sex, body mass index, education, and social support influence functional results after total knee arthroplasty. Geriatr Orthop Surg Rehabil. 2017:8:71-7.

24. McHugh GA, Campbell M, Luker KA. Predictors of outcomes of recovery following total hip replacement surgery: a prospective study. Bone Joint Res. 2013;2:248-54.

25. Szots $\mathrm{K}$, Konradsen $\mathrm{H}$, Solgaard S, Ostergaard B. Telephone follow-up by nurse after Total knee arthroplasty: results of a randomized clinical trial. Orthop Nurs. 2016;35:411-20

26. Lee YS, Laffrey SC. Predictors of physical activity in older adults with borderline hypertension. Nurs Res. 2006;55:110-20.

27. Nelson ME, Rejeski WJ, Blair SN, Duncan PW, Judge JO, King AC, et al. Physical activity and public health in older adults: recommendation from the American College of Sports Medicine and the American Heart Association. Circulation. 2007;1 16:1094-105.

28. Haskell WL, Lee IM, Pate RR, Powell KE, Blair SN, Franklin BA, et al. Physical activity and public health: updated recommendation for adults from the American College of Sports Medicine and the American Heart Association. Circulation. 2007;116:1081-93.

29. Resnick B, Spellbring AM. Understanding what motivates older adults to exercise. J Gerontol Nurs. 2000;26:34-42.

30. Lewis BA, Marcus BH, Pate RR, Dunn AL. Psychosocial mediators of physical activity behavior among adults and children. Am J Prev Med. 2002;23:26-35.

31. Trost SG, Owen N, Bauman AE, Sallis JF, Brown W. Correlates of adults' participation in physical activity: review and update. Med Sci Sports Exerc. 2002;34:1996-2001.

32. Peeters GM, Brown WJ, Burton NW. Psychosocial factors associated with increased physical activity in insufficiently active adults with arthritis. I Sci Med Sport. 2015;18:558-64.

33. Stubbs B, Hurley M, Smith $T$. What are the factors that influence physical activity participation in adults with knee and hip osteoarthritis? A systematic review of physical activity correlates. Clin Rehabil. 2015;29:80-94.

34. Dominick GM, Zeni JA, White DK. Association of psychosocial factors with physical activity and function after total knee replacement: an exploratory study. Arch Phys Med Rehabil. 2016;97:S218-25.

35. Waldrop D, Lightsey OR Jr, Ethington CA, Woemmel CA, Coke AL. Selfefficacy, optimism, health competence, and recovery from orthopedic surgery. J Couns Psychol. 2001;48:233-8.

36. Scult M, Haime V, Jacquart J, Takahashi J, Moscowitz B, Webster A, et al. A healthy aging program for older adults: effects on self-efficacy and morale. Adv Mind Body Med. 2015;29:26-33.

37. Liu N, Liu S, Yu N, Peng Y, Wen Y, Tang J et al. Correlations among Psychological Resilience, self-efficacy, and Negative Emotion in Acute Myocardial Infarction Patients after Percutaneous Coronary Intervention. Front Psychiatry. 2018;9:1.

38. Janiszewska M, Kulik T, Zolnierczuk-Kieliszek D, Drop B, Firlej E, Gajewska I. General self-efficacy level and health behaviours in women over the age of 45 years who have undergone osteoporosis treatment. Prz Menopauzalny. 2017;16:86-95. 
39. Lien R-Y, Wei J, Li J-Y, Tung H-H, Chen C-Y. Difference in predictors of self efficacy and compliance between diabetic and non diabetic patients who underwent coronary artery bypass surgery. The Journal of Nursing. 2012;59: 40-50.

40. Fitzgerald JD, Orav EJ, Lee TH, Marcantonio ER, Poss R, Goldman L, et al. Patient quality of life during the 12 months following joint replacement surgery. Arthritis Rheum. 2004;51:100-9.

41. Peak EL, Parvizi J, Ciminiello M, Purtill JJ, Sharkey PF, Hozack WJ, et al. The role of patient restrictions in reducing the prevalence of early dislocation following total hip arthroplasty: a randomized, prospective study. J Bone Joint Surg Am. 2005;87:247-53.

42. Peters A, Tijink M, Veldhuijzen A, Huis in 't Veld R. Reduced patient restrictions following total hip arthroplasty: study protocol for a randomized controlled trial. Trials. 2015;16:360.

43. van den Akker-Scheek I, Stevens M, Groothoff JW, Bulstra SK, Zijlstra W. Preoperative or postoperative self-efficacy: which is a better predictor of outcome after total hip or knee arthroplasty? Patient Educ Couns. 2007;66: 92-9.

44. Wylde V, Dixon S, Blom AW. The role of preoperative self-efficacy in predicting outcome after total knee replacement. Musculoskeletal Care. 2012;10:110-8.

45. Magklara $E$, Burton $C R$, Morrison V. Does self-efficacy influence recovery and well-being in osteoarthritis patients undergoing joint replacement? A systematic review. Clin Rehabil. 2014;28:835-46.

46. de Vries H, Kremers SP, Smeets T, Brug J, Eijmael K. The effectiveness of tailored feedback and action plans in an intervention addressing multiple health behaviors. Am J Health Promot. 2008;22:417-25.

47. Ayers DC, Franklin PD, Ring DC. The role of emotional health in functional outcomes after orthopaedic surgery: extending the biopsychosocial model to orthopaedics. AOA critical issues. J Bone Joint Surg Am. 2013;95:e165.

Ready to submit your research? Choose BMC and benefit from:

- fast, convenient online submission

- thorough peer review by experienced researchers in your field

- rapid publication on acceptance

- support for research data, including large and complex data types

- gold Open Access which fosters wider collaboration and increased citations

- maximum visibility for your research: over $100 \mathrm{M}$ website views per year

At BMC, research is always in progress.

Learn more biomedcentral.com/submissions 